\title{
Nuclear factor E2 related factor (NRF2) inhibits mast cell- mediated allergic inflammation via SIRT4-mediated mitochondrial metabolism
}

\author{
Binya $\mathrm{Hu}^{1}$, Sijun $\mathrm{Zhao}^{1}$, Min Huang ${ }^{1}$, Jihao Ren ${ }^{2}$ \\ ${ }^{1}$ Department of Otorhinolaryngology, Head and Neck Surgery, Hunan Children's Hospital, Changsha, China; ${ }^{2}$ Department of Otorhinolaryngology, \\ Head and Neck Surgery, Xiangya Hospital Central South University, Changsha, China \\ Contributions: (I) Conception and design: B Hu; (II) Administrative support: S Zhao, M Huang; (III) Provision of study materials or patients: B Hu; (IV) \\ Collection and assembly of data: B Hu, J Ren; (V) Data analysis and interpretation: B Hu; (VI) Manuscript writing: All authors; (VII) Final approval \\ of manuscript: All authors. \\ Correspondence to: Binya Hu; Sijun Zhao. Department of Otorhinolaryngology, Head and Neck Surgery, Hunan Children's Hospital, No.86 Ziyuan \\ Rd., Yuhua District, Changsha 410007, China. Email: zhizi628@126.com; Zhaosj3991@sohu.com.
}

\begin{abstract}
Background Mast cell (MC)-mediated inflammation is essential for allergic rhinitis, and nuclear factor E2 related factor (NRF2) is found to inhibit inflammation. This study investigated whether NRF2 could inhibit MC inflammation and its molecular mechanisms concerning SIRT4.

Methods: Real-time quantitative PCR (RT-qPCR) and western blot were used to detect gene expression, and Elisa kit was used to detect the content of histamine and inflammatory cytokines in the medium of MCs, and Seahorse XF instrument was used to measure the mitochondrial metabolism of MCs. Knockdown SIRT4 and establish SIRT4 overexpression of HMC-1 cells to study the function of SIRT4.

Results: As an activator of NRF2, 4-Octyl Itaconate increases not only NRF2 expression but also increases SIRT4 expression. Although 4-Octyl Itaconate could reduce the histamine release and degranulation of MCs, which was induced by compound 48/80, SIRT4 knockdown decreased the inhibition of 4-Octyl Itaconate. Similarly, 4-Octyl Itaconate inhibited the secretion of inflammatory cytokines (TNF- $\alpha$, IL-1 $\beta$, IL6, and IL-8) by MCs, which was induced by LPS, but SIRT4 knockdown decreases the inhibition of 4-Octyl Itaconate. Also, the up-regulation of SIRT4 significantly inhibited mitochondrial metabolism in MCs and inhibited SIRT1 and P-p65 protein expression after inducing by $100 \mathrm{ng} / \mathrm{mL}$ LPS for 1 hour.
\end{abstract}

Conclusions: NRF2 inhibits MC degranulation and MC-mediated inflammation by promoting SIRT4, and SIRT4 overexpression inhibits the mitochondrial metabolism of MCs.

Keywords: Nuclear factor E2 related factor (NRF2); SIRT4; mast cells (MC); allergic rhinitis; inflammation

Submitted Aug 10, 2020. Accepted for publication Nov 06, 2020.

doi: 10.21037/apm-20-1848

View this article at: http://dx.doi.org/10.21037/apm-20-1848

\section{Introduction}

Allergic rhinitis is a type I allergy that mediates sensitization of mast cells (MC) by IgE antibodies after exposure to an allergen in atopic individuals (1). Statistics show that the prevalence of allergic rhinitis in the United States is 5-22\%, and it costs about $\$ 7$ billion annually to treat allergic rhinitis (2). In China, the prevalence of allergic rhinitis also shows a significantly increasing trend; about 30 million people yearly (3). Although allergic rhinitis does not threaten the patient's life safety, a series of rhinitis clinical symptoms including nasal itching, stuffy nose, sneezing, and runny nose can seriously affect the patient's quality of life and cause personal and social-economic burden $(4,5)$.

MCs are cells derived from CD4+ spinal cord hematopoiesis, which enter the peripheral blood from the bone marrow and eventually migrate to the corresponding 
sites to differentiate and mature (6). MCs play an essential role in the pathogenesis of allergic rhinitis, and allergens specifically bind to sensitized MC surface FceRI, triggering degranulation of MCs, releasing inflammatory mediators and cytokines associated with type I allergies, causing vasodilatation, increased vascular permeability, and other inflammatory responses $(7,8)$. From the relationship between MCs and allergic rhinitis, inhibition of histamine and other related inflammatory cytokines by MCs can ease the symptoms of allergic rhinitis to some extent, which is one foundation for the development of drugs to treat allergic rhinitis (9).

Nuclear factor E2 related factor (NRF2) is an important transcription factor in the CNC family, which plays an antiinflammatory role in regulating inflammatory response(10). Previous studies have found that activation of NRF2 inhibits substance $\mathrm{P}$-induced $\mathrm{MC}$ activation and degranulation (11) and that the NRF2/HO-1 pathway is involved in regulating human MC-mediated allergic inflammation (12). However, previous studies only proved that NRF2 was involved in regulating the occurrence of allergic rhinitis and human MC-mediated allergic inflammation. The molecular mechanism of NRF2 in regulating inflammation in allergic rhinitis is still unclear. Whether NRF2 regulates MCmediated allergic inflammation by regulating mitochondrial metabolism, whether this is related to SIRT4 is also unknown. In this study, we found NRF2 upregulated SIRT4 expression, and upregulated SIRT4 inhibited mitochondrial metabolism and NF- $\kappa B$ signaling pathways, thereby inhibiting human MC-mediated allergic inflammation. We present the following article in accordance with the MDAR reporting checklist (available at http://dx.doi.org/10.21037/ apm-20-1848).

\section{Methods}

\section{Reagents and instruments}

4-Octyl Itaconate (HY-112675) was bought from MedChemExpress (USA). Lipopolysaccharide (LPS, 00-4976-03), RPMI-1640 medium (61870044), and Fetal bovine serum (FBS, 10437028) were bought from ThermoFisher SCIENTIFIC (USA). Antibody for NRF2 (ab137750), SIRT4 (ab10140), SIRT1 (ab110304), p65 (ab16502), P-p65 (ab86299), GAPDH (ab181602) and Elisa kit for histamine (ab213975), TNF- $\alpha$ (ab100654), IL-1 $\beta$ (ab46052), IL-6 (ab100712) and IL-8 (ab46032) were all purchased from ABCAM (UK). PrimeScript RT
Reagent Kit with gDNA Eraser (RR047B, Takara, Japan) and GoTaq ${ }^{\circledR}$ qPCR Master Mix (A6002, Promega, China). XFe24 extracellular flux assay kits (B35616) were bought from Agilent (USA). Nuclear and Cytoplasmic Protein Extraction Kit were bought from Sangon Biotech (China).

PCR instrument (S-1000, BIO-RAD, China), Fluorescence quantitative polymerase chain (FQD-96A, Hangzhou Bori Technology Co., Ltd., China), Extracellular Flux Analyzer (XFe-24, Agilent, USA), Microscope (DMI3000, Leica, German).

\section{Cell culture and transfection}

HMC-1 cells were purchased from the Shanghai Cell Bank of the Chinese Academy of Sciences and were cultured with RPMI- 1640 plus $10 \%$ FBS in $37^{\circ} \mathrm{C}$ and $5 \% \mathrm{CO}_{2}$.

Si-SIRT4 was synthesized by Sangon Biotech (China) and was directly transfected into cells with Lipofectamine ${ }^{\mathrm{TM}}$ 2000, and the cell could be used for research after si-SIRT4 transfer for 24 hours and verified by PCR and western blot. The SIRT4 overexpressing HMC-1 cell line was set up by Genmeditech and verified by PCR and western blot.

\section{Real-time quantitative PCR (RT-qPCR)}

Levels of miRNA/mRNA were detected by RT-qPCR as previously described (13). Reverse transcription program: $37^{\circ} \mathrm{C}-15$ min, $85^{\circ} \mathrm{C}-5$ second, $4{ }^{\circ} \mathrm{C}$-until the end; RTqPCR parameter settings: $95^{\circ} \mathrm{C}$ for 30 seconds, 40 cycles of $90{ }^{\circ} \mathrm{C}$ for 5 seconds, and $65^{\circ} \mathrm{C}$ for 30 seconds. PCR primer: NRF2-F:5'-TTCCCGGTCACATCGAGAG-3', NRF2-R: 5'-TCCTGTTGCATACCGTCTAAATC-3'; SIRT4-F: 5'-GAATCGGGGATACCAGACTACA-3', SIRT4-R: 5'-GCCAGCCTACGAAGTTTCTCG-3'; GAPDH-F: 5'-TAGCGGCTAGCGGTAT-3', GAPDH-R: 5'-CGGGGCTATGGCTAGCTAGCTTTC-3'.

\section{Western blot}

Protein levels were analyzed by the western blot, as described previously (13). The blocking solution was $5 \%$ TBST diluted skim milk powder and blocked at room temperature for 1 hour. The primary antibody [NRF2 (ab137750, 1:1,000), SIRT4 (ab10140, 1:500), SIRT1 (ab110304, 1:1,000), p65 (ab16502, 1:500), P-p65 (ab86299, 1:500), GAPDH (ab181602, 1:3,000)] was diluted with blocking solution and incubated for 2 hours at room temperature, and the secondary antibody was diluted with blocking solution 
and incubated for 1 hour at room temperature. Image $(\mathrm{NIH}$, USA) was used to analyze the gray value of the protein band, and GAPDH normalized the protein.

\section{Toluidine blue staining}

HMC-1 cells $\left(2 \times 10^{6}\right)$ were inoculated into a 6 -well plate. After drug treatment, the medium was removed, washed three times with $\mathrm{PBS}$, and $1 \mathrm{ml}$ of toluidine blue solution was added to each well. After staining for 30 seconds, the toluidine blue solution was discarded. The color separation was conducted for 10 seconds with a $95 \%$ ethanol solution, and then the ethanol solution was discarded and washed once with PBS. Remove the coverslip onto the slide, then observe and take a picture with a microscope.

\section{Mitochondrial metabolism}

Mitochondrial respiratory metabolism of MCs is assessed by Seahorse XF instruments, as previously described (14). Briefly, oxygen consumption rate (OCR) and spare respiratory capacity (SRC) were measured using XFe-24 Extracellular Flux Analyzer (Agilent, Santa Clara, CA, USA) and XFe24 extracellular flux assay kits (B35616, Agilent, Santa Clara, CA, USA) to test mitochondrial oxidative phosphorylation reaction according to the instructions. The final concentration of Oligomycin, FCCP, and Rotenone/ Antimycin A was 1.0, 0.5, and $0.5 \mu \mathrm{M}$, respectively. After the experiment was completed, collected the total protein extracted from each well and determined the concentration. We normalize the OCR/SCR values with protein concentration. In this study, wild-type and SRT4 overexpressing HMC-1 were collected after $100 \mathrm{ng} / \mathrm{mL}$ LPS stimulation for 1 hour to assess mitochondrial metabolism.

\section{Statistical analysis}

SPSS20.0 analyzed the data in its present study. Multiple groups were compared with the one-way ANOVA and the Duncan test as post hoc tests. And $\mathrm{P}<0.05$ means a significant difference.

\section{Results}

NRF2 activator, 4-Octyl Itaconate upregulated SIRT4 expression

4-Octyl Itaconate was the activator of NRF2 (15) and was added to the medium of MCs to upregulate NRF2 expression. As shown in Figure 1A, 4-Octyl Itaconate was found to be not significantly cytotoxic to MCs when the dose less than $200 \mu \mathrm{M}$, but $250 \mu \mathrm{M}$ 4-Octyl Itaconate was significantly toxic to MCs, so we chose $200 \mu \mathrm{M}$ as the working concentration in our study. Four hours after adding 4-Octyl Itaconate to the medium of MCs, we collected the cells and detected gene expression. We found (Figure 1B,C) as an NRF2 activator, 4-Octyl Itaconate could promote NRF2 mRNA and protein expression. 4-Octyl Itaconate also promotes SIRT4 gene expression.

\section{SIRT4 inhibits histamine release and degranulation of MCs}

Compound 48/80 promotes histamine release and degranulation in MCs, and inhibition of histamine release and degranulation from MCs is essential to treat allergic rhinitis. As shown in Figure 2A, 4-Octyl Itaconate could significantly upregulate SIRT4 expression in MCs with treatment by $48 / 80$, and si-SIRT4 reduced SIRT4 protein expression with treatment by $48 / 80$. Also, 4-Octyl Itaconate could significantly decrease the release of histamine (Figure $2 B$ ) and degranulation from MCs after inducing by 48/80 (Figure 2C), but si-SIRT4 increased them.

\section{SIRT4 inbibits LPS-induced inflammation in MCs}

TNF- $\alpha$, IL-1 $\beta$, IL-6, and IL-8 are common inflammatory cytokines and are essential for allergic rhinitis. We detect the content of TNF- $\alpha$, IL- $1 \beta$, IL- 6 and IL- 8 in the medium of MCs after inducing by LPS, and found that (Figure 3) 4-Octyl Itaconate could significantly decrease the content of TNF- $\alpha$, IL- $1 \beta$, IL- 6 and IL- 8 in the medium of MCs after inducing by LPS, but si-SIRT4 increased them.

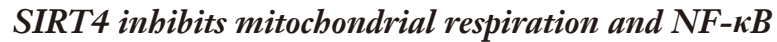 pathway in MCs}

Seahorse XF instrument was used to measure the mitochondrial metabolism of MCs, and we found (Figure 4A) upregulated SIRT4 could significantly decrease basal OCR and SRC of MCs, it showed that upregulated SIRT4 inhibited the mitochondrial metabolism of MCs. Upregulated SIRT4 decreased SIRT1 protein expression and P-p65 protein expression after inducing by $100 \mathrm{ng} / \mathrm{mL}$ LPS for one hour (Figure 4B,C). 
A
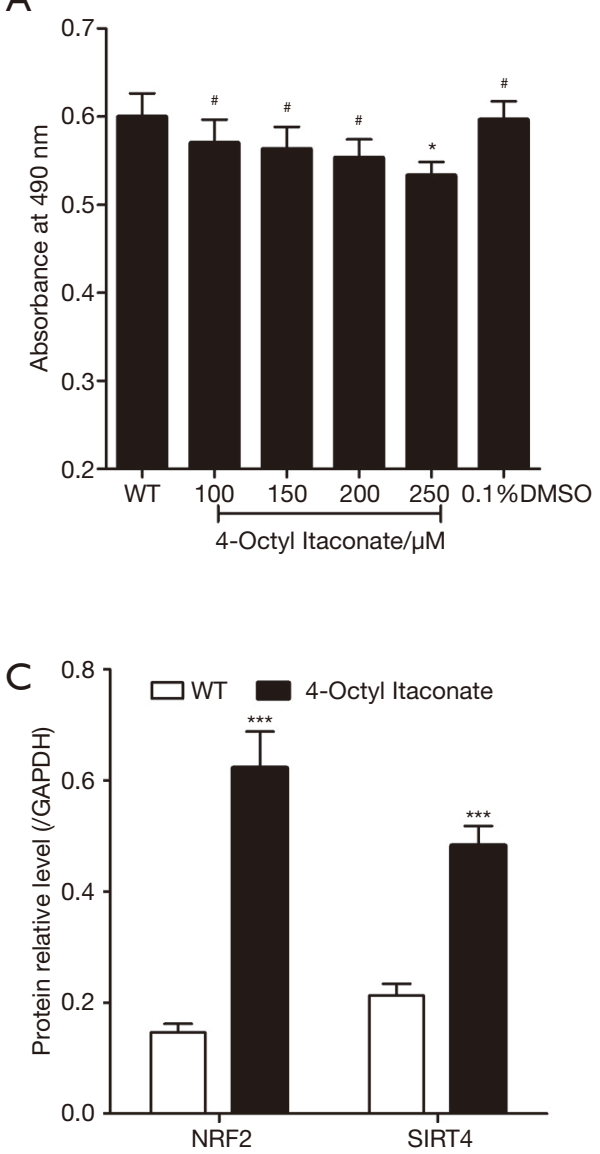

B
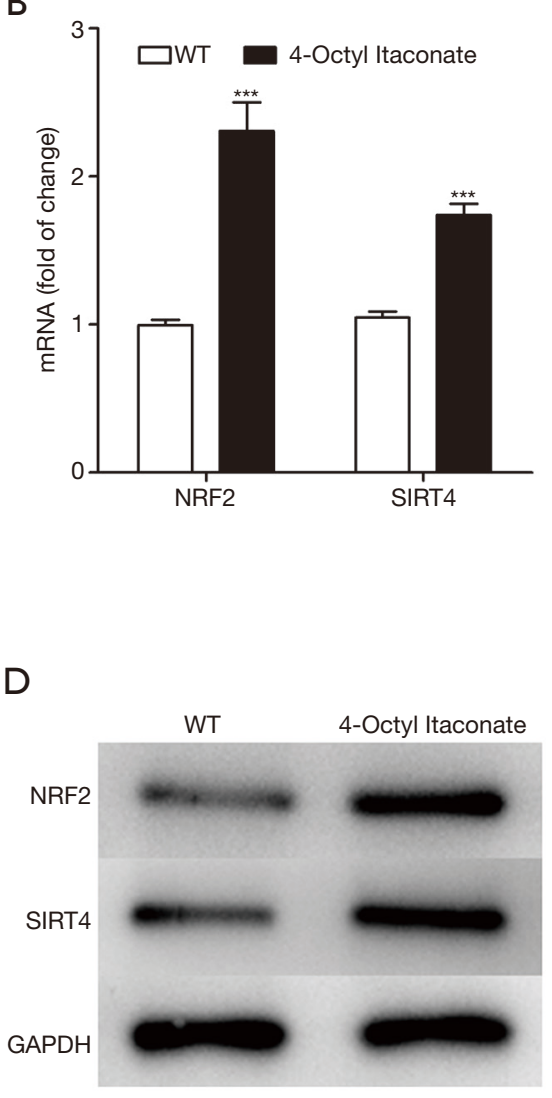

Figure $14-O c t y l$ Itaconate promotes NRF2 and SIRT4 expression in mast cells. (A) Cytotoxicity of 4-Octyl Itaconate on mast cells, which was measured by MTT, and compared OD490 values. (B) RT-qPCR detected NRF2 and SIRT4 mRNA in mast cells. (C) NRF2 and SIRT4 protein in mast cells was detected by western blot. Three independent repetitions per experiment. WT, wild type, it indicated HMC-1 cells with no treatment. 4-Octyl Itaconate indicated $200 \mu \mathrm{M}$ 4-Octyl Itaconate was added to HMC-1 cells for 4 hours. ", P>0.05 vs. WT group; *, $\mathrm{P}<0.05$; **, $\mathrm{P}<0.001$ vs. WT group. NRF2, nuclear factor E2 related factor; RT-qPCR, real-time quantitative PCR.

\section{Discussion}

NRF2 is a crucial transcription factor in the CNC family, is located at the $2 \mathrm{q} 31$ locus, and it has six different functional regions, named Neh1-Neh6, which are widely present in various tissues and organs of the body and interact with antioxidant elements ARE to activate downstream gene transcription further to regulate antioxidant and antiinflammatory proteins (16). Previous studies have found that exerts anti-inflammatory function in vitro and in vivo models $(17,18)$, such as NRF2 activated AMP-activated protein kinase (AMPK) can inhibit the expression of LPS-induced inflammatory genes by promoting nuclear transfer and phosphorylation of NRF2, thereby improving the survival rate of LPS-induced mice (18). In addition,
Lee et al. found that 3,4,5-Trihydroxycinnamic acid inhibits lipopolysaccharide (LPS)-induced inflammation by Nrf2 activation in vitro and improves survival of mice in LPSinduced endotoxemia model in vivo (19). And Qi et al. found that isorhamnetin could decrease the expression of PGE 2, NO, IL-6 and IL-8 in human gingival fibroblasts, but NRF2 knockdown reversed the anti-inflammatory effects of isorhamnetin, which indicated that NRF2 was necessary for isorhamnetin to inhibit inflammation in human gingival fibroblasts (20).

In this study, we found 4-Octyl Itaconate (15), a specific activator of NRF2, upregulated not only NRF2 in MCs but also upregulated SIRT4. SIRT4 is an essential member of the Sirtunin family of proteins localized to mammalian cell 


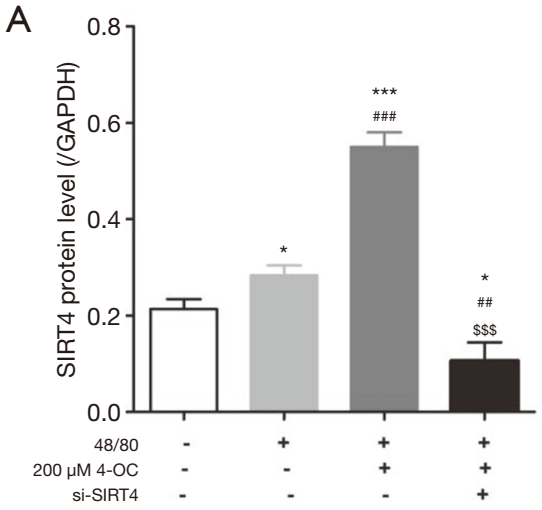

B

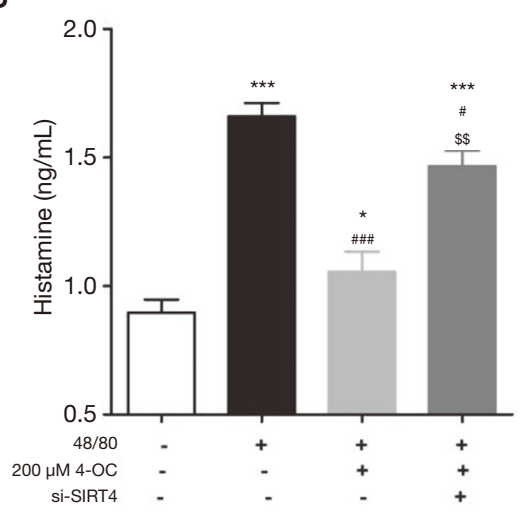

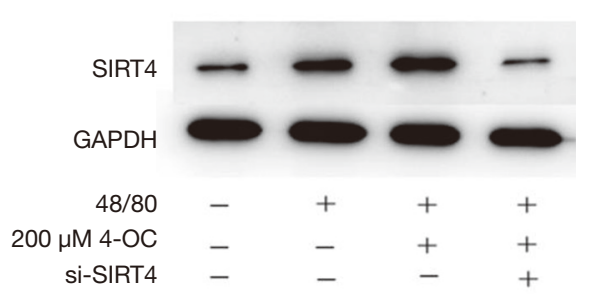

C

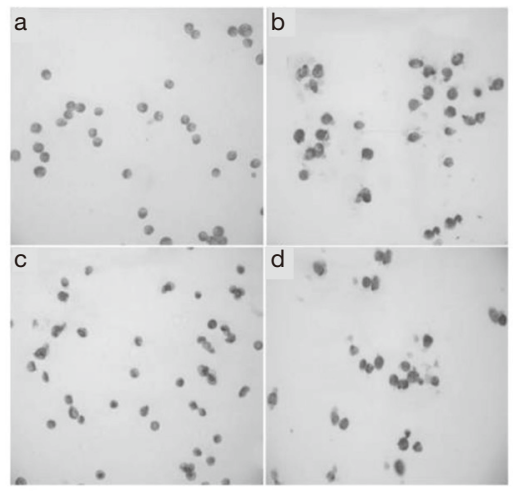

Figure 2 Effect of SIRT4 on histamine release and degranulation in mast cells. (A) SIRT4 protein expression in mast cells with different treatments. (B) The content of histamine in the medium of mast cells with different treatments. (C) Toluidine blue staining was used to observe mast cell degranulation, and (a) (WT) was an HMC-1 cell without any treatment (100x); (b) (48/80) was 10 $\mu \mathrm{g} / \mathrm{mL}$ compound 48/80 was added to HMC-1 cell culture medium for 2 hours; (c) (4-OC) was $10 \mu \mathrm{g} / \mathrm{mL}$ compound 48/80 was added to HMC-1 cell culture medium for 2 hours after adding $200 \mu \mathrm{M}$ 4-Octyl Itaconate for 4 hours; (d) Si-SIRT4 was $10 \mu \mathrm{g} / \mathrm{mL}$, and the compound 48/80 was added to the HMC-1 cell culture medium for 2 hours after transferring to si-SIRT4 for 24 hours. Three independent repetitions per experiment. *, $\mathrm{P}<0.05$ and ${ }^{* * *}, \mathrm{P}<0.001$ vs. WT group; ${ }^{\#}, \mathrm{P}<0.05 ;^{\# \#}, \mathrm{P}<0.01 ;{ }^{\# \# \#}, \mathrm{P}<0.001$ vs. $48 / 80$ groups; ${ }^{\$ \$}, \mathrm{P}<0.01 ;{ }^{\$ \$ \$}, \mathrm{P}<0.001$ vs. 4-OC group.

mitochondria. Previous studies have shown that SIRT1, SIRT3, and SIRT6, members of the Sirtunin family, are being found to be involved in regulating inflammatory responses by sensing the NAD/NADH energy axis $(14,21)$. Although there are few studies on regulating SIRT4 and inflammation, SIRT4 can inhibit amino acids, fatty acid catabolism, and promote fatty acid anabolism by catalyzing the deacetylation, deglutamylation, ADP ribosylation, lipidation or regulation of substrate expression of substrates, thereby regulating the biological effects of glucose tolerance and insulin antagonism (22). Therefore, we hypothesized that NRF2 might regulate the cellular energy supply by upregulating SIRT4 and then taking part in regulating the inflammatory response.

MC degranulation refers to a functional state of MCs. Their high-affinity FceR1 binding sensitizes MCs to allergen-specific IgE. When the same allergen reappears and crosslinks cell surface IgE, FceR1 activates MCs via signal transduction and releases intragranular active medium in the granules to induce type I hypersensitivity $(23,24)$. Previous studies have shown that $(25,26)$ allergens act on MCs to activate and release the active mediator is the direct cause of allergic/allergic reactions, while compound $48 / 80$ is a tool for degranulation of MCs. We found NRF2 inhibited compound 48/80-induced MC degranulation and histamine release, but this inhibitory effect was significantly 
A

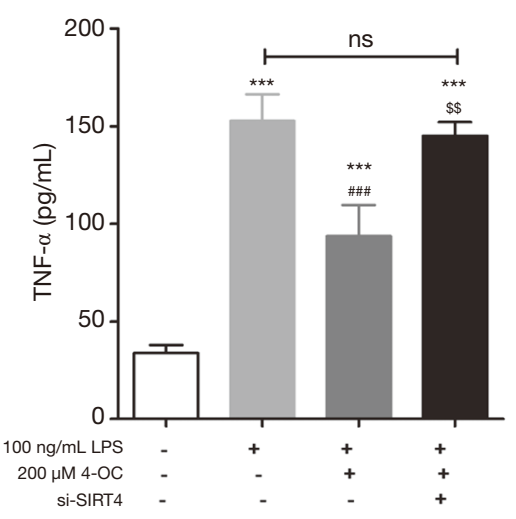

C

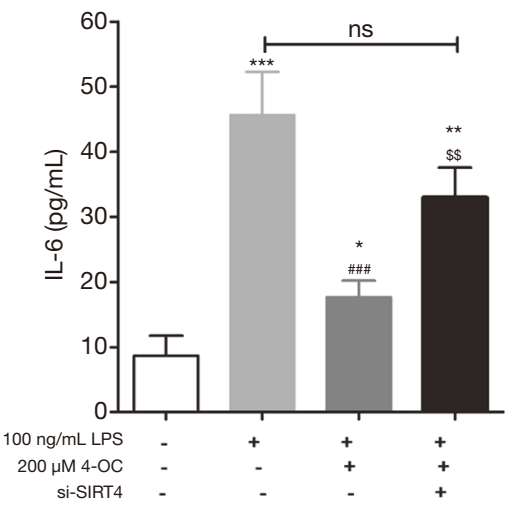

B

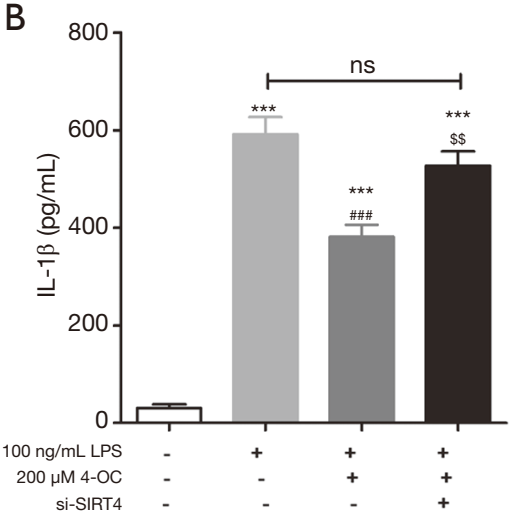

D

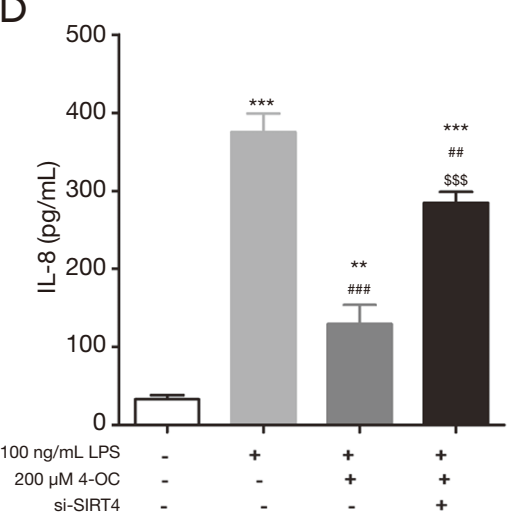

Figure 3 Effect of SIRT4 on the expression of inflammatory cytokines induced by LPS in mast cells. (A,B,C,D) The content of TNF- $\alpha$ (A), IL-1 (B), IL-6 (C), and IL-8 (D). Three independent repetitions per experiment. *, $\mathrm{P}<0.05$, **, $\mathrm{P}<0.01$ and ${ }^{* * *}, \mathrm{P}<0.001$ vs. WT group; ${ }^{\# \#}$, $\mathrm{P}<0.01 ;{ }^{\# \#,}, \mathrm{P}<0.001$ vs. LPS group; ${ }^{\text {\$s }}, \mathrm{P}<0.01,{ }^{\text {\$\$S }}, \mathrm{P}<0.001$ vs. 4-OC group.
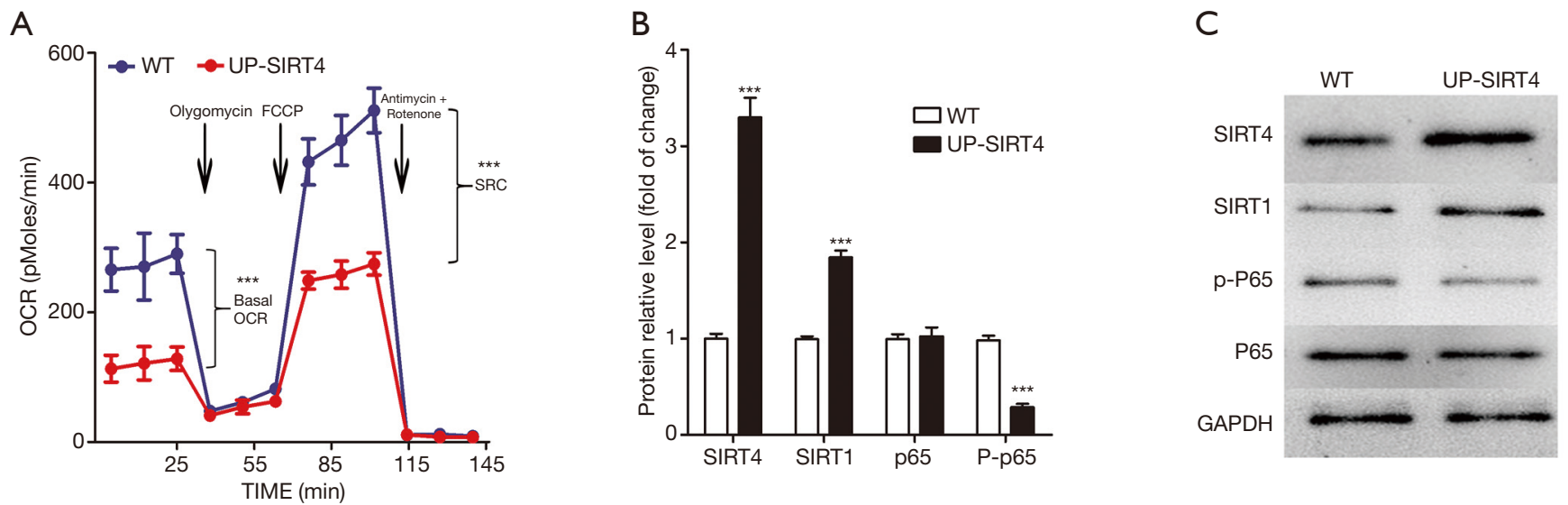

Figure 4 Effects of SIRT4 expression on mitochondrial metabolism and NF- $\kappa$ B pathway in mast cells. (A) Seahorse XF instrument was used to measure the mitochondrial metabolism in different HMC-1 cells after inducing by $100 \mathrm{ng} / \mathrm{mL}$ LPS for 1 hour. (B) SIRT4, SIRT1, p65, and P-p65 protein were detected in HMC-1 cells by western blot after inducing by $100 \mathrm{ng} / \mathrm{mL}$ LPS for 1 hour. Three independent repetitions per experiment. ${ }^{* *}, \mathrm{P}<0.001$ vs. WT group. 
reduced in SIRT4 knockdown MCs. These findings showed that SIRT4 functioned as a downstream protein of NRF2 and was involved in MC degranulation by regulating NRF2.

LPS is a significant component in the cell wall of Gramnegative bacteria and can induce inflammation in the body tissues and is one of the primary pathogens of inflammatory diseases in the body. And previous studies have shown that lipopolysaccharide-induced inflammatory responses in cells can establish in vitro cellular inflammatory models $(27,28)$. In this study, we used LPS to stimulate MCs to produce an inflammatory response to set up an in vitro cellular inflammatory model. We found NRF2 inhibited LPS-induced MC-mediated allergic inflammation, but this inhibition was significantly reduced in SIRT4 knockdown MCs. These findings suggested that SIRT4 functioned as a downstream protein of NRF2 involved in regulating MC-mediated allergic inflammation by NRF2. Previous studies have shown that many intermediate proteins and cellular inflammatory factors need to be synthesized during the inflammatory process, which required an abundant supply of energy $(29,30)$, and SIRT1, SIRT3, and SIRT6 be involved in regulating inflammation by regulating cellular mitochondrial metabolism (14,21). And SIRT4 was found mainly regulated glutamine metabolism in mitochondria, and glutamine was the primary source of the supplemental tricarboxylic acid cycle which as a raw material for $\alpha$-ketoglutaric (31), so SIRT4 was an "engine" that provided energy support for cells to perform various biological functions $(32,33)$. Therefore, SIRT4, a protein localized to mitochondria, is also suspected to be involved in regulating MC-mediated allergic inflammation by regulating mitochondrial metabolism.

We showed a SIRT4 overexpressing MC line. We found that the up-regulation of SIRT4 significantly reduced the base OCR and SRC of a MC, suggesting that the up-regulation of SIRT4 has an inhibitory effect on mitochondrial metabolism. As we have already mentioned above, the main function of SIRT4 discovered so far times is to regulate glutamine metabolism in mitochondria, and glutamine as raw material of $\alpha$-oxoglutarate is the main source for supplementing the TCA cycle (34), and is an "engine" that provides energy support for inflammation (35). Study showed that the loss of SIRT4 resulted in the dysregulation of mammalian target of rapamycin complex 1 (mTORC1) expression and loss of stability for cAMP responsive element binding2 (CREB2), thereby increasing GDH activity and glutamine metabolism (36). Glutamine is a metabolite necessary for synthesis and secretion of inflammatory factors, and synthesis and secretion of inflammatory factors is one of characteristics of tumor cell metabolism $(35,36)$. Also, we found that SIRT4 overexpression could significantly increase SIRT1 expression and inhibit p65 protein phosphorylation. SIRT1 (silent information regulator 1 ) is a histone deacetylase widely expressed in human cells. It carried out important biological functions by deacetylating multiple transcription factors, including p53 (37), UCP2 (38), P300 (39)

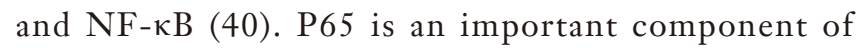
$\mathrm{NF}-\kappa \mathrm{B}$, which only functions after it is acetylated. In inflammatory responses, SIRT1 deacetylated p65, thus inhibiting the transcription of TNF- $\alpha$, IL- 6 , and other inflammatory genes downstream of NF- $\mathrm{KB}$ (40) p65, is an important protein in the TLR/NF- $\kappa \mathrm{B}$ signaling pathway. Its phosphorylation-mediated translocation (from the cytoplasm to the nucleus) is an important marker of the activation of $\mathrm{NF}-\kappa \mathrm{B}$ signaling. NF- $\kappa \mathrm{B}$, also known as a $\kappa$-binding nuclear factor, belonged to the Rel protein family and was an important signal transduction molecule involved in the inflammatory reaction (41).

\section{Conclusions}

Overexpression of NRF2 inhibits MC degranulation and MC-mediated inflammation by upregulating the expression of SIRT4 in MCs in vitro, and SIRT4 overexpression inhibits mitochondrial metabolism of MCs. NRF2 may be a potential target to treat allergic rhinitis by inhibiting MC mitochondrial metabolism.

\section{Acknowledgments}

Funding: None.

\section{Footnote}

Reporting Checklist: The authors have completed the MDAR reporting checklist. Available at http://dx.doi.org/10.21037/ apm-20-1848

Data Sharing Statement: Available at http://dx.doi. org/10.21037/apm-20-1848

Conflicts of Interest: All authors have completed the ICMJE uniform disclosure form (available at http://dx.doi. org/10.21037/apm-20-1848). The authors have no conflicts of interest to declare. 
Ethical Statement: The authors are accountable for all aspects of the work in ensuring that questions related to the accuracy or integrity of any part of the work are appropriately investigated and resolved.

Open Access Statement: This is an Open Access article distributed in accordance with the Creative Commons Attribution-NonCommercial-NoDerivs 4.0 International License (CC BY-NC-ND 4.0), which permits the noncommercial replication and distribution of the article with the strict proviso that no changes or edits are made and the original work is properly cited (including links to both the formal publication through the relevant DOI and the license). See: https://creativecommons.org/licenses/by-nc-nd/4.0/.

\section{References}

1. Small P, Keith PK, Kim H. Allergic rhinitis. Allergy Asthma Clin Immunol 2018;14:51.

2. Schatz M. A survey of the burden of allergic rhinitis in the USA. Allergy 2007;62 Suppl 85:9-16.

3. Zhang L, Zhang Y, Desrosiers M, et al. Genetic association study of FOXP3 polymorphisms in allergic rhinitis in a Chinese population. Hum Immunol 2009;70:930-4.

4. Hanahan D, Weinberg RA. The hallmarks of cancer. Cell 2000;100:57-70.

5. Tabatabaian F, Casale TB. Allergic rhinitis management: what's next? Expert Rev Clin Immunol 2018;14:191-6.

6. Siebenhaar F, Redegeld FA, Bischoff SC, et al. Mast Cells as Drivers of Disease and Therapeutic Targets. Trends Immunol 2018;39:151-62.

7. Shiraishi Y, Jia Y, Domenico J, et al. Sequential engagement of FceRI on Mast Cells and Basophil Histamine H(4) Receptor and FceRI in Allergic Rhinitis. J Immunol 2013;190:539-48.

8. Bui TT, Piao CH, Song CH, et al. Skullcapflavone II attenuates ovalbumin-induced allergic rhinitis through the blocking of Th2 cytokine production and mast cell histamine release. Int Immunopharmacol 2017;52:77-84.

9. Howarth $\mathrm{PH}$. The cellular basis for allergic rhinitis. Allergy 1995;50:6-10.

10. Kim J, Cha YN, Surh YJ. A protective role of nuclear factor-erythroid 2-related factor-2 (Nrf2) in inflammatory disorders. Mutat Res 2010;690:12-23.

11. Jadkauskaite L, Bahri R, Farjo N, et al. Nuclear factor (erythroid-derived 2)-like-2 pathway modulates substance $\mathrm{P}$-induced human mast cell activation and degranulation in the hair follicle. J Allergy Clin
Immunol 2018;142:1331-3.e8.

12. Ye J, Piao H, Jiang J, et al. Polydatin inhibits mast cellmediated allergic inflammation by targeting PI3K/ Akt, MAPK, NF-kB and Nrf2/HO-1 pathways. Sci Rep 2017;7:11895.

13. Tao J, Zhang J, Ling Y, et al. Mitochondrial Sirtuin 4 Resolves Immune Tolerance in Monocytes by Rebalancing Glycolysis and Glucose Oxidation Homeostasis. Front Immunol 2018;9:419.

14. Liu TF, Vachharajani V, Millet P, et al. Sequential actions of SIRT1-RELB-SIRT3 coordinate nuclearmitochondrial communication during immunometabolic adaptation to acute inflammation and sepsis. J Biol Chem 2015;290:396-408.

15. Mills EL, Ryan DG, Prag HA, et al. Itaconate is an antiinflammatory metabolite that activates Nrf2 via alkylation of KEAP1. Nature 2018;556:113-7.

16. Sandberg M, Patil J, D'Angelo B, et al. NRF2-regulation in brain health and disease: implication of cerebral inflammation. Neuropharmacology 2014;79:298-306.

17. Koh K, Kim J, Jang YJ, et al. Transcription factor Nrf2 suppresses LPS-induced hyperactivation of BV-2 microglial cells. J Neuroimmunol 2011;233:160-7.

18. Mo C, Wang L, Zhang J, et al. The crosstalk between Nrf2 and AMPK signal pathways is important for the anti-inflammatory effect of berberine in LPS-stimulated macrophages and endotoxin-shocked mice. Antioxid Redox Signal 2014;20:574-88.

19. Lee JW, Bae CJ, Choi YJ, et al. 3,4,5-trihydroxycinnamic acid inhibits lipopolysaccharide (LPS)-induced inflammation by Nrf2 activation in vitro and improves survival of mice in LPS-induced endotoxemia model in vivo. Mol Cell Biochem 2014;390:143-53.

20. Qi F, Sun JH, Yan JQ, et al. Anti-inflammatory effects of isorhamnetin on LPS-stimulated human gingival fibroblasts by activating Nrf2 signaling pathway. Microb Pathog 2018;120:37-41.

21. Liu TF, Vachharajani VT, Yoza BK, et al. NAD+dependent sirtuin 1 and 6 proteins coordinate a switch from glucose to fatty acid oxidation during the acute inflammatory response. J Biol Chem 2012;287:25758-69.

22. Kim EA, Yang SJ, Choi SY, et al. Inhibition of glutamate dehydrogenase and insulin secretion by KHG26377 does not involve ADP-ribosylation by SIRT4 or deacetylation by SIRT3. BMB Rep 2012;45:458-63.

23. Schwartz SL, Cleyrat C, Olah MJ, et al. Differential mast cell outcomes are sensitive to FceRI-Syk binding kinetics. Mol Biol Cell 2017;28:3397-414. 
24. Li X, Park SJ, Jin F, et al. Tanshinone IIA suppresses FceRI-mediated mast cell signaling and anaphylaxis by activation of the Sirt1/LKB1/AMPK pathway. Biochem Pharmacol 2018;152:362-72.

25. Wu Z, Macneil AJ, Junkins R, et al. Mast cell FceRIinduced early growth response 2 regulates $\mathrm{CC}$ chemokine ligand 1-dependent CD4+ T cell migration. J Immunol 2013;190:4500-7.

26. Handlogten MW, Kiziltepe T, Alves NJ, et al. Synthetic allergen design reveals the significance of moderate affinity epitopes in mast cell degranulation. ACS Chem Biol 2012;7:1796-801.

27. Shen B, Zhao C, Chen C, et al. Picroside II Protects Rat Lung and A549 Cell Against LPS-Induced Inflammation by the NF-кB Pathway. Inflammation 2017;40:752-61.

28. Lee JH, Moon JH, Lee YJ, et al. SIRT1, a Class III Histone Deacetylase, Regulates LPS-Induced Inflammation in Human Keratinocytes and Mediates the Anti-Inflammatory Effects of Hinokitiol. J Invest Dermatol 2017;137:1257-66.

29. Stofkova A. Leptin and adiponectin: from energy and metabolic dysbalance to inflammation and autoimmunity. Endocr Regul 2009;43:157-68.

30. Pyper SR, Viswakarma N, Yu S, et al. PPARalpha: energy combustion, hypolipidemia, inflammation and cancer. Nucl Recept Signal 2010;8:e002.

31. Lakhter AJ, Hamilton J, Konger RL, et al. Glucoseindependent Acetate Metabolism Promotes Melanoma Cell Survival and Tumor Growth. J Biol Chem 2016;291:21869-79.

32. Bolzoni M, Chiu M, Accardi F, et al. Dependence on glutamine uptake and glutamine addiction characterize myeloma cells: a new attractive target. Blood

Cite this article as: $\mathrm{Hu} \mathrm{B}$, Zhao S, Huang M, Ren J. Nuclear factor E2 related factor (NRF2) inhibits mast cell-mediated allergic inflammation via SIRT4-mediated mitochondrial metabolism. Ann Palliat Med 2020;9(6):3839-3847. doi: 10.21037/ apm-20-1848
2016;128:667-79.

33. Son J, Lyssiotis CA, Ying H, et al. Glutamine supports pancreatic cancer growth through a KRAS-regulated metabolic pathway. Nature 2013;496:101-5.

34. Le A, Lane AN, Hamaker M, et al. Glucose-independent glutamine metabolism via TCA cycling for proliferation and survival in B cells. Cell Metab 2012;15:110-21.

35. Qiu JL, Song BL, Wang YJ, et al. Role of glutamine in the mediation of E-cadherin, p120-catenin and inflammation in ventilator-induced lung injury. Chin Med J (Engl) 2018;131:804-12.

36. Csibi A, Fendt SM, Li C, et al. The mTORC1 pathway stimulates glutamine metabolism and cell proliferation by repressing SIRT4. Cell 2013;153:840-54.

37. Lai M, Du G, Shi R, et al. MiR-34a inhibits migration and invasion by regulating the SIRT1/p53 pathway in human SW480 cells. Mol Med Rep 2015;11:3301-7.

38. Li X, Xie Z, Lin M, et al. Renalase protects the cardiomyocytes of Sprague-Dawley rats against ischemia and reperfusion injury by reducing myocardial cell necrosis and apoptosis. Kidney Blood Press Res 2015;40:215-22.

39. Kim EK, Choi EJ. Compromised MAPK signaling in human diseases: an update. Arch Toxicol 2015;89:867-82.

40. Wagner SA, Satpathy S, Beli P, et al. SPATA2 links CYLD to the TNF- $\alpha$ receptor signaling complex and modulates the receptor signaling outcomes. EMBO J 2016;35:1868-84.

41. Wullaert A, Bonnet MC, Pasparakis M. NF- $\kappa$ B in the regulation of epithelial homeostasis and inflammation. Cell Res 2011;21:146-58.

(English Language Editor: J. Chapnick) 